\title{
Cadeia cinética aberta e fechada: uma reflexão crítica
}

\author{
Open and closed kinetic chain: a critical reflection
}

\author{
Auristela Duarte de Lima Moser ${ }^{[a]}$, Mariane França Malucelli ${ }^{[b]}$, Sandra Novaes Bueno ${ }^{[b]}$ \\ [a] Programa de Pós-Graduação em Tecnologia em Saúde da Pontifícia Universidade Católica do Paraná (PPGTS/PUCPR), Curitiba, \\ PR - Brasil, e-mail: auristela.lima@pucpr.br \\ [b] Fisioterapeuta, Universidade Positivo (UP), Curitiba, PR - Brasil.
}

\section{Resumo}

Introdução: Desde 1973, quando Steidler procedeu à transposição dos princípios de cadeia cinética aberta e fechada da mecânica para a reabilitação, muitos estudos têm sido feitos sobre as consequências dos exercícios envolvendo tais cadeias, mas pouco tem se estudado sobre a validade de tal definição, seus benefícios e riscos. A comunidade da reabilitação associou a definição de CCA e CCF a alguns exemplos clássicos de exercícios, sem questionar se os componentes envolvidos na definição eram suficientes para estabelecer este conceito. Método: As autoras realizaram uma revisão bibliográfica que incluiu artigos com o conceito de cadeia cinética aberta e fechada e livros de cinesiologia, mecânica e dinâmica, buscando aproximações e divergências na definição e nos conceitos. Resultados: Na mecânica as cadeias abordadas são cinemáticas e não cinéticas e a transposição desses conceitos para a reabilitação foi literal, favorecendo o uso dos termos como sinônimos, mesmo existindo uma diferença entre eles: a cadeia cinemática não considera as forças causadoras do movimento ou do equilíbrio, já a cadeia cinética as considera. O termo cadeia cinética aberta não é mencionado na mecânica. Conclusões: Todos os exercícios envolvendo apenas uma articulação deveriam ser chamados exercícios isolados e o termo cadeia cinética fechada deveria ser dividido em três categorias: cadeia cinemática fechada, cadeia cinemática restrita e cadeia cinemática, concordando com o grau de liberdade de cada cadeia. Sugere-se que esses termos deveriam ser usados para descrever exercícios de múltiplas articulações, concordando com o grau de liberdade de cada exercício.

Palavras-chave: Cadeia cinética aberta. Cadeia cinética fechada. Cadeia cinemática. Funcionalidade. Reabilitação do LCA. 


\begin{abstract}
Introduction: Since 1973 when Steindler transcribed the principles of closed and open kinetic chain from the mechanics to the rehabilitation world, many studies have been conducted about the consequences of the exercises involving both types of kinetic chains, however there hasn't been a lot of studies concerning the validity about its definition, its benefits and risks. The community rehabilitation have associated the definition of closed and open kinetic chain, to some classic exercises without questioning if the components involved in definition was enough to establish such concept. Method: Authors have made a bibliography revision that included articles with the definition of kinetic chains, books of kinesiology, dynamic and mechanics searching a correlation some approximations and disagreements in the definition and concepts. Results: The mechanics chains mentioned are the kinematic chains and not kinetic chains and the transposition of this concepts to the rehabilitation was literary, favoring the use of terms as synonyms, even existing a difference between them: the kinematic chain doesn't consider the causing forces of movement or balance, however the kinetic chain has considered them. The term open kinematic chain is not mentioned in mechanics. Conclusions: All the exercises involving only one joint should be called isolated exercises and the term closed kinematic chain should be divided into three categories: closed kinematic chain, restrained kinematic chain and kinematic chain, in accordance to the degree of freedom of each chain. It is suggested that these terms should be used to describe multi joint exercises in accordance to the degree of freedom of each exercise.
\end{abstract}

Keywords: Closed kinetic chain. Open kinetic chain. Kinematic chain. Functionality. ACL rehabilitation.

\title{
Introdução
}

Os termos cadeia cinética aberta e cadeia cinética fechada vêm sendo muito usados nos últimos 15 anos pela comunidade da reabilitação. Neste período muitas pesquisas têm sido realizadas sobre o tema, a maioria envolvendo os exercícios para membros inferiores na reabilitação do ligamento cruzado anterior (LCA) (1-3).

A definição comumente utilizada para classificar exercícios em cadeia cinética é a de que, se o segmento distal estiver livre para se mover no espaço e não sustentar o peso corporal, a cadeia é considerada aberta, e na ocorrência do contrário a cadeia é considerada fechada $(3,4)$.

O assunto tomou grandes proporções em meados dos anos 90 , quando foram criados os protocolos envolvendo exclusivamente exercícios em cadeia cinética fechada na reabilitação das lesões de LCA $(3,5,6)$.

Graças à importância destes conceitos e sua repercussão nas práticas da reabilitação, observa-se que sua utilização, aliada a várias práticas fisioterapêuticas, é feita sem que haja discussão crítica sobre as decisões clínicas. Pode-se afirmar que não é o equipamento que potencialmente pode lesar o paciente, e sim o clínico utilizando o equipamento. $\mathrm{O}$ uso correto e criterioso evitará a deformação e prejuízos á reconstrução do LCA (7).

Todo tratamento possui riscos e benefícios e cabe ao terapeuta ser capaz de identificá-los e analisálos criticamente para melhor seleção dos recursos e o modo de utilizá-los. Para que isto aconteça é necessário que se tenha pleno conhecimento das bases teóricas dos conceitos que sustentam as práticas terapêuticas. A discussão, portanto, poderá favorecer uma reflexão sobre estes conceitos subsidiando a tomada de decisões clínicas e terapêuticas e aumentando a resolutibilidade das práticas.

\section{Metodologia}

Procedeu-se a uma revisão de literatura a partir das bases de dados Medline e Pubmed, selecionando ao fim 19 artigos, sendo alguns clássicos e outros mais contemporâneos. Foram fontes de pesquisa também 15 livros de mecânica, dinâmica, mecânica das máquinas e cinesiologia.

Os artigos foram captados de jornais de medicina desportiva e fisioterapia, incluindo o American Journal of Sports Medicine, Journal of Sports and Physical Therapy, Psysical Therapy, Journal of Sports Rehabilitation e 
Sports Medicine. Foram utilizadas as palavras-chave cadeia cinética, cadeia cinemática, cadeia cinética aberta, cadeia cinética fechada, forças de cisalhamento, reabilitação do joelho.

Incluíram-se todos os artigos que traziam a definição de cadeia cinética fechada e aberta, e mais quatro artigos escolhidos aleatoriamente que apesar de não trazerem a definição, citavam exemplos de exercícios envolvendo cadeias cinéticas a título de ilustração. Foram excluídos 15 artigos captados inicialmente que apesar de abordarem o tema, não traziam o conceito de cadeia cinética aberta e fechada.

Procedeu-se a uma leitura reflexiva das fontes, buscando-se o conceito de cadeia cinética implícito ou explícito neles, bem como as concordâncias e divergências encontradas nas descrições de exercícios utilizados na reabilitação com base nestas cadeias. Esta triangulação de dados possibilitou a análise crítica exposta a seguir. Os dados coletados foram sintetizados em um quadro exposto a seguir. A reflexão crítica baseou-se em uma análise de conteúdo, de modo a extrair termos-chave que agrupados levaram ao estabelecimento de categorias de discussão apresentadas no tópico de resultados. Estas categorias foram elencadas pela sua importância no processo de reflexão para o estabelecimento de práticas diagnósticas e terapêuticas.

\section{Resultados}

Quadro 1 - Resumo dos conceitos utilizados sobre CCA e CCF

(Continua)

\begin{tabular}{|c|c|c|}
\hline Autor & Cadeia cinética fechada & Cadeia cinética aberta \\
\hline $\begin{array}{l}\text { - Fitzgerald GK (1997) } \\
\text { - Bynum B (1995) } \\
\text { - Yack J (1993) } \\
\text { - Hening CE (1985) } \\
\text { - Cohen ZA (2001) }\end{array}$ & $\begin{array}{l}\text { Sustentam o peso corporal. É necessário movimento } \\
\text { em várias articulações para que se complete o } \\
\text { movimento, o segmento distal é normalmente fixo a } \\
\text { uma superfície sustentadora, e a resistência pode ser } \\
\text { aplicada tanto distal quanto proximal. }\end{array}$ & $\begin{array}{l}\text { Não sustentam o peso corporal, com } \\
\text { movimento ocorrendo em uma única } \\
\text { articulação. O segmento distal é livre para } \\
\text { se mover, e a resistência é normalmente } \\
\text { aplicada no segmento distal. }\end{array}$ \\
\hline - Bynum EB (1995) & $\begin{array}{l}\text { São seguros e eficazes e oferecem vantagens importantes } \\
\text { sobre exercícios em cadeia cinética aberta. }\end{array}$ & \\
\hline $\begin{array}{l}\text { - Henning CE } \\
\text { - Yack HJ }\end{array}$ & $\begin{array}{l}\text { Exercícios que sustentam o peso corporal causam } \\
\text { menos deformação no LCA. }\end{array}$ & $\begin{array}{l}\text { Exercícios que não sustentam o peso } \\
\text { corporal causam mais deformação no LCA. }\end{array}$ \\
\hline - Prentice WE (1991) & $\begin{array}{l}\text { O pé está normalmente em contato com o solo, o que } \\
\text { faz ser mais funcional. }\end{array}$ & \\
\hline - Palmitier AR (1994) & $\begin{array}{l}\text { Exercícios como o agachamento devem ser chamados } \\
\text { simplesmente de exercícios em cadeia cinética. }\end{array}$ & $\begin{array}{l}\text { Durante a extensão de perna sentada, } \\
\text { a força é aplicada perpendicular à tíbia, } \\
\text { causando um momento em flexão somente } \\
\text { no joelho. Tal isolamento da articulação } \\
\text { durante o exercício não tira vantagem } \\
\text { dos efeitos estabilizadores secundários de } \\
\text { outros músculos na cadeia cinética inferior. } \\
\text { Estes exercícios devem ser chamados } \\
\text { simplesmente exercícios isolados. }\end{array}$ \\
\hline - Davies & $\begin{array}{l}\text { Em uma cadeia cinética, cada segmento que se move } \\
\text { transmite forças para todos os outros segmentos, } \\
\text { afetando o movimento destes segmentos, e tem o seu } \\
\text { movimento influenciado pelas forças transmitidas por } \\
\text { outros segmentos (interdependência). }\end{array}$ & Articulação isolada. \\
\hline
\end{tabular}


Quadro 1 - Resumo dos conceitos utilizados sobre CCA e CCF

(Conclusão)

\begin{tabular}{|c|c|c|}
\hline Autor & Cadeia cinética fechada & Cadeia cinética aberta \\
\hline $\begin{array}{l}\text { - Steindler } \\
\text { - Relaux }\end{array}$ & $\begin{array}{l}\text { Isto cria um sistema em que o movimento em uma } \\
\text { articulação produz movimento em todas as outras } \\
\text { articulações de uma maneira previsível. O segmento } \\
\text { distal da articulação é fixo (pode apresentar-se } \\
\text { estacionário ou móvel) e suporta considerável } \\
\text { resistência externa, o que impede ou reduz seu } \\
\text { movimento livre. }\end{array}$ & $\begin{array}{l}\text { Caracteriza-se pelo segmento distal, que } \\
\text { termina livre no espaço. }\end{array}$ \\
\hline $\begin{array}{l}\text { - Waldrom } \\
\text { - Mabie }\end{array}$ & $\begin{array}{l}\text { Uma cadeia cinemática é aquela em que os elos e } \\
\text { juntas formam um ou mais circuitos fechados. Cada } \\
\text { circuito é um laço em que cada elo é conectado a pelo } \\
\text { menos dois outros elos. Se estes elos são conectados } \\
\text { de uma maneira em que nenhum movimento é } \\
\text { possível, o resultado é uma cadeia (estrutura) fixa } \\
\text { ou fechada. Uma cadeia restrita é alcançada quando } \\
\text { os elos estão conectados de tal maneira que, não } \\
\text { importam quantos ciclos de movimento passam através } \\
\text { dos elos, o movimento relativo será sempre o mesmo } \\
\text { entre os elos. Também é possível conectar os elos de } \\
\text { maneira que resultará em uma cadeia não restrita, o que } \\
\text { significa que o padrão de movimento varia de tempo } \\
\text { em tempo, dependendo da quantidade de fricção } \\
\text { presente nas juntas. Se um elo de uma cadeia restrita se } \\
\text { torna fixo, o resultado então é um mecanismo. }\end{array}$ & $\begin{array}{l}\text { Não consta este termo nos livros de } \\
\text { mecânica. }\end{array}$ \\
\hline
\end{tabular}

\section{A gênese do conceito}

A análise revela que o conceito de cadeia cinética passou a ser utilizado a partir de 1980, quando Reulaux lançou o conceito de elos rígidos e juntas na engenharia mecânica e construção de máquinas, e classificou também cadeia cinemática e mecanismo.

Cadeia Cinemática é definida como uma montagem de elos e articulações interconectadas de maneira que promova um movimento controlado em resposta a um movimento fornecido como estímulo.

Mecanismo é definido como uma cadeia cinemática na qual pelo menos um elo foi fixado, ou conectado, à moldura de referência, a qual pode estar em movimento.

Desde 1973, relaciona-se o conceito de cadeia cinemática com a biomecânica, afirmando-se que o corpo humano pode ser visto como um sistema de elos rígidos interconectados por articulações, e que o comportamento articular e recrutamento muscular muda mais quando a extremidade encontra resistência do que quando está livre. Posteriormente, esta afirmação foi corroborada em outros estudos $(8,9)$.

Utilizados inicialmente, os termos cadeia cinética aberta e fechada tiveram como objetivo prover um esquema de classificação que diferenciava dois enfoques distintos de exercícios: o de cadeia cinética fechada, quando o segmento distal encontrava resistência, sendo a ação muscular e a função articular diferentes de quando o segmento distal era livre para se mover denominado de cadeia cinética aberta.

Assim, uma cadeia cinética aberta caracteriza-se pela liberdade do segmento distal, ao passo que na cadeia cinética fechada o segmento distal da articulação é fixo e suporta uma considerável resistência externa, o que impede ou reduz sua liberdade de movimentação. Eventualmente a resistência interna poderá ser superada, 
e nesse caso, a porção distal da articulação movimentar-se-á contra essa resistência (10,11). A partir da definição original, alguns novos termos apareceram baseados em tal conceito.

O fato de Steindler não ter afirmado que o segmento distal deveria estar estático, mas que se este superasse a resistência poderia movimentar-se contra esta, abriu possibilidades para que outros exercícios se enquadrassem neste conceito. Nesse caso, não somente o fato de não ser estático passou a ser fundamental, mas também o de que os exercícios em CCF podem ter seu grau de liberdade reduzido e não somente seu movimento impedido - como os exercícios até hoje estudados - passou a ter grande importância no conceito de CCF.

$\mathrm{Na}$ Cinesiologia mais esclarecimentos foram veiculados sobre os termos cadeia cinética fechada e aberta e cadeia cinemática. Veicula-se que as cadeias cinemáticas se compõem de uma série de ligações rígidas que são interconectadas por uma série de juntas centradas por um pino (12). Na engenharia, o sistema de juntas e ligações é construído de tal forma que o movimento de uma ligação produzirá movimento de todas as juntas de modo previsível. As cadeias cinemáticas da engenharia formam um sistema fechado, ou cadeia cinemática fechada. Esta definição baseada na engenharia trás um novo componente, a interdependência e a previsibilidade dos movimentos envolvidos no sistema, formando assim um sistema fechado. Quando analisamos os exercícios ora citados e comparamos com esta definição, vemos que o agachamento, considerado cadeia cinética fechada, não forma um sistema fechado, pois a extremidade do tronco está livre para se mover no espaço. Então o agachamento não deveria ser classificado como um exercício em cadeia cinética fechada.

Numa discussão mais contemporânea questiona-se sobre o sistema de elos fechados e a existência de exercícios em cadeia cinética fechada pura, afirmando que os exercícios devem ser combinações dos dois tipos de cadeia $(13,14)$.

Tanto Norkin quanto Andrews usaram a mesma referência para seus estudos individuais, o livro Cinesiologia de Brunnstrom (15), que considera como a principal característica de uma cadeia cinemática aberta a liberdade do segmento distal no espaço, enquanto que na cadeia cinemática fechada o segmento distal é fixo. Aparentemente isto não constitui novidade, mas ele também afirma que "cadeias cinemáticas fechadas são comumente usadas em máquinas; e cadeias cinemáticas abertas são mais comuns no corpo humano, como exemplificada na coluna vertebral e nos membros". Porém são encontradas algumas cadeias fechadas também no corpo humano, como o cinturão pélvico em que os segmentos estão unidos por duas articulações sacras ilíacas, a sínfise púbica e a caixa torácica, em que cada costela com sua conexão vertebral e esternal forma um anel.

Analisando tais afirmações e comparando com a engenharia é correto afirmar que somente alguns exemplos de cadeia fechada são encontrados no corpo humano, pois realmente formam um sistema fechado. Analisando a anatomia humana verificamos que outro exemplo se enquadra nesta definição: a articulação temporomandibular, que é fechada em ambos os lados, havendo interdependência quanto ao movimento e as forças que o determinam.

Nos livros de Cinesiologia encontra-se também o conceito de cadeia cinemática trazido por Steindler para a reabilitação, mudando a denominação para cadeia cinética. A distinção de cinemática e cinética pode contribuir para verificar se a diferença entre os dois é relevante e se traz alguma alteração à definição original.

Diferencia-se cinética e cinemática quando se afirma que a cinemática ocupa-se apenas dos aspectos geométricos do movimento enquanto que a cinética analisa as forças causadoras (16).

A definição de cadeia cinemática trazida por livros de mecânica não considera as forças envolvidas na produção dos movimentos, aceitando como cadeia cinemática qualquer arranjo de elos rígidos conectados por juntas. Os elos e juntas formam um ou mais circuitos fechados e cada circuito fechado é um laço em que cada elo é conectado a pelo menos dois outros elos (17). Quando um número de elos é conectado em pares, o resultado é uma cadeia cinemática. Se estes elos são conectados de uma maneira em que nenhum movimento é possível, o resultado é uma cadeia (estrutura) fixa ou fechada (18).

Considera-se uma cadeia restrita quando os elos estão conectados de tal modo que independentemente de quantos ciclos de movimento passam através deles, o movimento relativo será sempre o mesmo entre os elos. Também é possível conectar os elos de maneira que resulte em uma cadeia não restrita, o que significa que o padrão de movimento varia de tempos em tempos dependendo da quantidade de fricção presente nas juntas. Quando o elo de uma cadeia restrita se torna fixo, o resultado então é um mecanismo (18). 


\section{Questionando a funcionalidade como benefício dentro das cadeias cinéticas fechadas}

A análise de alguns estudos evidencia, além de divergências sobre a definição de cadeia cinética fechada e aberta, os riscos e benefícios de cada tipo de cadeia. Alguns autores afirmam que os exercícios em cadeia cinética aberta não são funcionais, argumentando que exercícios deste tipo não sustentam o peso corporal, e têm seu movimento ocorrendo em uma única articulação pelo fato do segmento distal ser livre para se mover.

Como os exercícios em cadeia cinética fechada sustentam o peso corporal, são necessários movimentos em várias articulações para que se complete o movimento global, e o segmento distal é normalmente fixo a uma superfície sustentadora (4). Novamente defende-se que o grupo dos exercícios em CCF se refere a exercícios que sustentam o peso corporal (5) e, já que o pé está normalmente em contato com o solo, atividades que fazem uso deste sistema fechado são mais funcionais (2). Observa-se, assim, forte tendência a estabelecer que exercícios em cadeia cinética fechada tendam a promover mais função do que os exercícios em cadeia cinética aberta, porque envolvem primariamente atividades de sustentação do peso do corpo $(1,2-5)$.

Existem inúmeros exercícios para o fortalecimento dos músculos que cruzam a articulação do joelho, mas após os muitos estudos envolvendo a recuperação do LCA, parece existir uma tendência na comunidade da reabilitação em usar a classificação de exercícios em cadeia cinética aberta e fechada para dividir dois tipos de exercícios em dois grupos principais, tornando comum o estabelecimento da relação entre o nome do exercício e a sua classificação, evidenciado em citações como: "durante dois tipos de exercícios: 1. extensão de joelho resistida, um exercício em cadeia aberta; e 2., o agachamento paralelo, um exercício em CCF”(6); ou ainda "exercícios em CCA, cadeira extensora de perna e exercício em CCF, o agachamento" (7).

Tal relação surgiu do fato de que a maioria dos estudos feitos utilizou somente dois exercícios, um para cada tipo de cadeia cinética, e esta situação favoreceu generalizações sobre a funcionalidade de cada tipo de cadeia, pois o único exercício observado para se estudar cadeia cinética fechada foi o agachamento, que sustenta o peso corporal, e o único exercício observado para se estudar cadeia cinética aberta foi a cadeira extensora sem sustentação de peso. Generalizou-se que CCA não é funcional e CCF é funcional sem se ter realizado uma análise de outros exercícios que se enquadram no mesmo tipo de cadeia cinética. Uma breve reflexão sobre o conceito de funcionalidade ancorada na literatura nos remete a autores que compartilham a afirmação de que a funcionalidade é a capacidade de manter as habilidades físicas e mentais necessárias a uma vida independente e autônoma (19).

Esta visão é compartilhada também por outros pesquisadores que realçam o conceito de funcionalidade em sua dimensão cotidiana, sendo esta uma medida da independência e autonomia individual relacionada à aptidão para reproduzir no dia a dia os movimentos básicos articulares necessários ao atendimento das necessidades cotidianas (20).

No que se refere ao membro superior, a flexão ou a abdução ativa do ombro é um exercício em cadeia cinética aberta e muito funcional para alcançar o que necessitamos em nosso dia a dia, ao contrário do leg press, que é um exercício em cadeia cinética fechada e não necessariamente funcional, pois não encontramos nas atividades de vida diária situações em que a resistência venha de encontro ao membro inferior como o aparelho leg press realiza.

Além da funcionalidade, outro componente polêmico na aplicação das cadeias abertas e fechadas é o cisalhamento, como fator de risco no processo terapêutico, objeto do próximo tópico.

\section{Questionando o cisalhamento como risco dentro das cadeias cinéticas abertas}

Uma força pode ser decomposta em componentes que são perpendiculares e paralelos a uma alavanca. Deve-se notar que os componentes que se encontram em cada um desses dois planos têm efeito diferente sobre o eixo em torno do qual a alavanca gira (uma tem efeito de rotação e a outra, de translação). De fato, qualquer força pode ser vista não somente como atuando através de uma articulação, mas também entre dois objetos que mantenham contato. Quando a força que está sobre um objeto é criada pelo contato com outro 
objeto, o componente da força que se mantém perpendicular às superfícies de contato pode ser chamado de força de contato ou força normal. O componente que se mantém paralelo com as superfícies de contato é conhecido como força de cisalhamento, ou de deslocamento, se ela está pronta para causar movimento entre as duas superfícies.

Dependendo da natureza das superfícies de contato, uma força de cisalhamento pura pode resultar em força de atrito em sentido contrário entre as duas superfícies. A força de cisalhamento pode ser equilibrada por um deslocamento oposto do componente perpendicular da gravidade, pois isto cria um torque flexor em torno do eixo da articulação do joelho, pela fricção que ocorre entre as superfícies articulares, ou pela força de compressão criada em ligamentos articulares tensos. A maioria dos ligamentos articulares está posicionada não somente para resistir à distração da articulação, mas também para resistir às forças de cisalhamento (14).

Decorrente dos resultados das pesquisas que mostram as limitações de se usar exercícios em CCA, como o aumento da força de cisalhamento na cartilagem articular e a translação anterior da tíbia (21-24), e do exercício não ser considerado funcional, muitos terapeutas abandonaram o uso deste tipo de exercício, desprezando benefícios, como o acesso ao déficit muscular individual. A reabilitação deve incluir o acesso individual dos músculos fracos ou debilitados para que sejam fortalecidos individualmente, pois se forem colocados dentro de uma cadeia cinética ainda fracos, a musculatura proximal e distal vai compensar e mascarar o déficit do músculo debilitado dentro do sistema, não fortalecendo o que está realmente debilitado e incentivando padrões compensatórios que afetarão toda a cadeia.

Nesta perspectiva, é necessário também avaliar as limitações dos exercícios uniarticulares considerados CCA, como no exemplo da extensão de joelho, no qual a translação da tíbia pode ocorrer quando a sobrecarga é posicionada distalmente no membro e o movimento é realizado de maneira lenta. Sendo assim, devem-se identificar as limitações de qualquer situação dentro da reabilitação e alterá-la adequadamente, em vez de descartá-la sem se prender a conceitos limitantes. Neste mesmo exemplo, a diminuição do braço de resistência com a aproximação do ponto de aplicação da carga em relação à articulação e redução da velocidade do exercício diminuiria significativamente a translação tibial $(25,26)$. Ainda com relação ao mesmo exemplo, sabe-se que limitando a amplitude de movimento do joelho para extensão, iniciando em $100^{\circ}$ de flexão e finalizando a $30^{\circ}$, protege-se o LCA de estresse desnecessário.

A partir destas constatações, pode-se argumentar que não é o tipo de exercício que pode trazer danos ao paciente, mas a maneira como o terapeuta utiliza o exercício ou o equipamento.

Analisando os estudos feitos sobre o aumento de cisalhamento nos exercícios uniarticulares ou CCA, percebe-se que o único exercício testado foi a extensão de joelho resistida, e a conclusão divulgada parece ter levado a uma generalização de que todos os exercícios em CCA fazem isto, e não que este exercício específico causa mais força de cisalhamento em LCA. A gênese dessas afirmações e contraponto ao exemplo anterior é o célebre exercício em cadeia cinética fechada conhecido como agachamento, no qual se verifica que a força de cisalhamento em LCA é menor, por causa da cocontração dos isquiotibiais e da compressão do peso corporal $(27,28)$. A conclusão veiculada em tais estudos é de que os exercícios em CCA são prejudiciais para a reabilitação do LCA e os exercícios em CCF são benéficos.

Esta generalização também traz alguns problemas, quando fazemos a análise de forças vetoriais. A análise de exercícios individualmente no padrão CCF pode apontar forças de cisalhamento maiores do que as compressivas. Tome-se como exemplo o hack squat, um exercício em cadeia cinética fechada que limita a possibilidade de manipular o movimento e o estresse do quadril e joelho de maneira segura, por não permitir alterar a posição do quadril relativa à linha de força. Quando o quadril fica restrito no movimento posterior, a pelve empurra o encosto, que retorna às forças impostas, causando uma reação à ação imposta. Estas forças de reação anteriores irão aumentar significativamente as forças de cisalhamento no joelho, mesmo que a tíbia permaneça perpendicular ao apoio do pé, na posição fletida.

Sendo assim, acredita-se na importância de analisar cada exercício individualmente, de acordo com a o quadro cínico e funcional, para que se possam identificar os riscos e benefícios e assim fazer a melhor escolha para o paciente. Com base nessa afirmação, pode-se exemplificar que enquanto o aumento de força de cisalhamento no joelho é fator limitante para a reabilitação do LCA, o mesmo não ocorre em patologias 
degenerativas como a artrose no joelho, quando é preferível utilizar exercícios que exerçam maior cisalhamento e menor compressão, em função da vulnerabilidade da matriz óssea.

\section{Uma nova proposta}

Introduzida por Steindler e derivada de Releaux, a terminologia cadeia cinética aberta e fechada descreve o mesmo conceito de cadeia cinemática e elos rígidos trazidos da mecânica, em que o termo cadeia cinemática indica uma combinação de vários segmentos rígidos conectados por juntas interpostas. O sistema é considerado fechado se ambas as extremidades são conectadas a uma moldura fixa, assim prevenindo translação tanto da junta distal quanto da proximal, criando um sistema em que movimento em uma junta produzirá movimento em todas as outras juntas de maneira previsível $(29,30)$.

Cadeia cinemática é um conceito biomecânico que inclui descrição de movimento, não levando em consideração as forças que os produzem. Steindler popularizou o termo cadeia cinética em referência ao mesmo conceito, porém cadeia cinética também é um conceito biomecânico, mas que considera as forças produtoras tanto de movimento quanto de equilíbrio. Concluímos então que os termos têm definições distintas, mas são normalmente usados como sinônimos na literatura da reabilitação.

Seguindo este mesmo pensamento, Davies (9) refere que em uma cadeia cinética cada segmento que se move transmite forças a todos os outros segmentos, afetando o movimento destes segmentos, e tendo seu movimento influenciado por forças transmitidas por outros segmentos, e isto é chamado de interdependência, dentro da mecânica.

Analisando a afirmação anterior e comparando com todas as descrições da mecânica, o termo cadeia cinética aberta deixa de ser preciso, pois se o movimento ocorrer em somente uma articulação, é quase impossível que este influencie o movimento em outros segmentos, pois somente este se move. Então sugerimos que este seja chamado somente de movimento uniarticular ou movimento isolado (11).

Chamar exercícios em cadeia cinética aberta de movimentos uniarticulares torna possível aplicar a descrição mecânica de Mabie à biomecânica, dividindo as cadeias cinéticas (movimentos multiarticulares) desta maneira: cadeia cinética fechada, cadeia cinética restrita e cadeia cinética (menos restrita), de acordo com a liberdade permitida em cada cadeia.

A partir do conceito anterior, pode-se exemplificar a utilização desta nova classificação nos exercícios. Uma cadeia cinética fechada caracteriza-se por ter suas duas extremidades fixas (não necessariamente estáticas), obtendo assim um movimento totalmente previsível, e pode ser exemplificada com o leg press ou o hack squat, em que quatro segmentos estão envolvidos no movimento (o tronco, o pé, a coxa e a perna) e os dois segmentos mais distantes estão fixos (tronco e pé).

Cadeia cinética restrita caracteriza-se por ter uma de suas extremidades fixa e a outra restrita, que pode ser guiada ou ter qualquer outro tipo de restrição. Quando um segmento é guiado ou restrito, pode-se prever o trajeto que este realizará, mas não o exato movimento dos segmentos envolvidos, tornando este menos previsível que o anterior, podendo ser exemplificado com o agachamento na Smith Machine, em que os mesmos segmentos estão envolvidos, e um segmento é fixo (o pé) e o outro é guiado por uma barra fixa ao trilho de deslocamento (o tronco).

Finalmente se tem a cadeia cinética, que passa a ser caracterizada por movimentos multiarticulares (11), com uma extremidade fixa e a outra livre, assim não sendo previsível. Esta pode ser exemplificada com o agachamento livre, no qual os mesmos quatro segmentos estão envolvidos, mas somente um está fixo e nenhum guiado, aumentando sensivelmente o grau de liberdade da cadeia.

A relevância de se distinguir os diferentes tipos de cadeia é que, ao se restringir o grau de liberdade de movimento, influencia-se o recrutamento de proprioceptores. Isto quer dizer que quanto menos restrito for o exercício, mais proprioceptores serão recrutados, mais estabilização dinâmica estará envolvida e, consequentemente, menor será a carga que poderá ser utilizada com segurança. E ainda quanto mais restrito for o exercício, menos estabilização dinâmica estará envolvida, levando a menor recrutamento de proprioceptores. Isto conduz a maior possibilidade de utilização de sobrecarga com segurança. 


\section{Conclusão}

Com base na pesquisa realizada, entende-se que a classificação que determina a cadeia cinética fechada e a cadeia cinética aberta não é suficientemente clara. Os argumentos usados para estabelecer esta definição não têm a consistência necessária para sustentar o conceito.

As fontes pesquisadas se referem à cadeia cinética fechada e aberta, porém não deixam claro como é feita esta classificação e qual o critério de escolha dos exercícios utilizados para se realizar as pesquisas; do mesmo modo, os resultados não apresentam dados consistentes sobre se é a ação da cadeia cinética aberta que causa deformação no LCA ou o exercício específico utilizado. Em virtude dessas inconsistências, é precipitado afirmar que apenas as cadeias cinéticas fechadas são funcionais, ou que somente os exercícios em cadeia cinética aberta causam excesso de cisalhamento com prejuízos ao sistema articular, como alguns desses artigos afirmam.

Após confronto com os conceitos da mecânica, sugere-se que a classificação seja complementada com os termos cadeia cinética fechada, cadeia cinética restrita e cadeia cinética. Também sugerimos que o termo cadeia cinética aberta seja substituído por movimento uniarticular ou isolado. Acredita-se que estas opções de terminologia trarão subsídios para melhor escolha do tipo de exercício a ser utilizado em diferentes momentos de cada da recuperação, individualmente e com segurança.

\section{Referências}

1. Shelbourne KD, Nitz P. Accelerated rehabilitation after anterior cruciate ligament reconstruction. J Orthop Sports Phys Ther. 1992;15(6):256-64.

2. Prentice WE. Closed kinecit chain exercise. In: Prentice WE, editor. Rehabilitation Techniques in Sports Medicine. 2nd ed. St Louis: Mosby; 1994. p. 98-107.

3. Hening CE, Lych MA, Glick JR. An in vivo strain gauge study of elongation of the anterior cruciate ligament. Am J Sports Med. 1985;13(1):22-6.

4. Jurist KA, Otis JC, Fitzgerald GK. Anteroposterior tibiofemoral displacements during Open versus closed kinetic chain exercises: issues in rehabilitation after anterior cruciate ligament reconstructive surgery. Phys Ther. 1997;77:1747-54.

5. Bynum BE, Barrack RL, Alexandre AH. Open versus closed chain kinetic exercises after anterior cruciate ligament reconstruction. Am J Sports Med. 1995;23(4):401-6.

6. Yack HJ, Collins CE, Whieldon TJ. Comparison of closed and open kinetic chain exercise in the anterior cruciate ligament-deficient knee. Am J Sports Med. 1993;21(1):49-54.

7. Cohen ZA, Roglic H, Grelsamer RP, Henry JH, Levine WN, Mow VC, et al. Patellofemoral stresses during open and closed kinetic chain exercises: an analysis using computer simulation. Am J Sports Med. 2001;29(4):480-7.

8. Haupental A, Santos DP. Força e contato patelofemoral como fundamentos biomecânicos para reabilitação da síndrome patelofemoral. Fisioter Mov. 2006;19(4):11-16.

9. Davies GJ. The need for critical thinking in rehabilitation. J. Sports Rehabilitation J Sport Rehabil. 1995;4(1):1-22.

10. Steindler A. Kinesiology of the human body under normal and pathological conditions. Springfield, IL: Charles C. Thomas; 1973.

11. Palmitier RA, An KN, Scott SG, Chao EYS. Kinetic chain exercise in knee reabilitation. Sports Medicine. 1991;11(6):402-13.

12. Smidt GL. Current open and closed kinetic chain concepts: clarifying or confusing. JOSPT. 1994;20(5):235. 
13. Andrews HW. Reabilitação física das lesões desportivas. 2a ed. Rio de Janeiro: Guanabara Koogan; 2000.

14. Levangie PK, Norkin CC. Joint structure and function: a comprehensive analysis. Philadelphia: FA Davis Company, 1992.

15. Smith LK, Weiss EL, Lehmkuhl LD. Cinesiologia clínica de Brunnstrom. 5a ed. São Paulo: Manole; 1997.

16. Hibbeler RC. Mecânica dinâmica. 8a ed. Rio de Janeiro: Ed. Abdr; 1999.

17. Waldron KJ, Kinzel GL. Kinematics, dynamics, and design of machinery. New York: John Wiley e Sons; 1999.

18. Mabie HH, Reinholtz CF. Mechanisms and dynamics of machinery. 4th ed. New York: John Wiley; 1986.

19. World Health Organization. Disability prevention and rehabilitation. Geneva: WHO; 1981.

20. Farias N, Buchalla CM. The international classification of functioning, disability and health: concepts, uses and perspectives. Rev Bras Epidemiol. 2005;8(2):187-93.

21. Grood ES, Suntay WJ, Noyes FR, Butler DL. Biomechanics of the knee-extension exercise. J. Bone Joint Surg. 1984;66(5):725-34.

22. Jurist KA. Isometric extension efforts. Am J Sports Med. 1985;13(4):254-8.

23. Lutz GE, Palmitier RA, An KN, Chao EYS. Comparison of tibiofemoral joint forces during open-kinetic-chain and closed-kinetic-chain exercises. J Bone Joint Surg Am. 1993;75(5):732-9.

24. Renstrom P, Arms SW, Stanwyck TS, Johnson RJ, Pope MH. Strain within the anterior cruciate ligament during hamstring and quadriceps activity. Am J Sports Med. 1986;14(1):83-7.

25. Baratta R, Solomonow M, Zhou BH. Muscular coactivation: the role of the antagonist musculature in maintaining knee stability. Am J Sports Med. 1988;16(2):113-22.

26. Draganich LF, Jaeger RJ, Knalj AR. Coactivation of the hamstrings and quadriceps during extension of the knee. J Bone Joint Surg Am. 1989;71(7):1075-81.

27. Kaufman KR. Mathematical model of knee forces during exercise [thesis]. Grand Forks: North Dakota State University; Grand Forks ND; 1988.

28. Solomonow M, Baratta R, Zhou BH. The synergistic action of the anterior cruciate liogament and thigh muscles in maiataining joint stability. Am J Sports Med. 1987;15(3):207-13.

29. Gowitzke BA, Horris M. Scientific basis of human movement. 3rd ed. Baltimore: Willians \& Wilkins; 1988.

30. Deusinger RH. Biomechanics in clinical practice. Phys Ther. 1984;64(12):1860-8.

Recebido: 23/03/2010

Received: 03/23/2010

Aprovado: 25/08/2010

Approved: 08/25/2010

Fisioter Mov. 2010 out/dez;23(4):641-50 